\title{
CONCENTRATION OF HOST BLOOD PROTEIN DURING FEEDING BY ANOPHELINE MOSQUITOES (DIPTERA: CULICIDAE) ${ }^{1}$
}

\author{
Hans Briegel and Licia Rezzonico
}

\begin{abstract}
During blood feeding, Anopheles stephensi ingested 2 to $10 \mu \mathrm{l}$ of blood. It took such large meals by releasing large amounts of a red rectal fluid as it fed. Although the fluid contained intact erythrocytes, the female was able to concentrate the dietary protein in the midgut by a factor of about 2 . Two larger species, An. albimanus and An. quadrimaculatus, consuming larger meals, released a clear rectal fluid and also concentrated their blood meals without losing protein. Therefore, gravimetric determinations of blood consumption would be misleading in Anopheles. Concentration of blood proteins during the act of feeding, before diuresis, is unique for Anopheles and led to increased fecundity when compared to blood meals given by enema. Similar to results with Aedes, fecundity was significantly lower with human than with guinea pig blood. It was further demonstrated that female Anopheles actually refed on successive days when a host was offered. In contrast to Aedes, subsequent blood meals produced an increase in the number of maturing oocytes.
\end{abstract}

Despite their important role as primary vectors of plasmodia causing primate malaria, female Anopheles have received comparatively little attention from insect physiologists, possibly because aedine and culicine mosquitoes are more easily reared and handled. Aedine and culicine mosquitoes generally require 1 blood meal for each gonotrophic cycle, while anopheline mosquitoes in the laboratory are routinely fed at least twice per gonotrophic cycle, sometimes even daily (Gerberg 1970, Horsfall 1972). To our knowledge, however, the physiological implications of these feeding regimes have never been analyzed.

As in many hematophagous insects, e.g., Rhodnius (Maddrell 1964) and Glossina (Gee 1975), a conspicuous diuresis begins in mosquitoes shortly after ingestion of blood (Boorman 1960, Stobbart 1977, Jones \& Brandt 1981, Williams et al. 1983). Nijhout \& Carrow (1978) have investigated diuresis in Anopheles freeborni Aitken and ascribed its control to a diuretic hormone. In all these studies the experimental analysis of diuresis began with cessation of blood feeding and continued for roughly $1 \mathrm{~h}$ thereafter. In Anopheles, however, excretion of

1. This project was supported by grants from the Swiss $\mathrm{Na}$ tional Science Foundation.

2. Department of Zoology, University of Zürich, $\mathrm{CH}-8057$ Zürich, Switzerland. rectal fluid starts a few seconds after piercing the skin of the host and ingestion of blood. A notable occurrence during the feeding of colonies of $A n$. stephensi Liston is the excretion of a bright red fluid. For convenience we will call these excretions rectal fluid, because their origin has not been investigated. Excretion of rectal fluid during feeding is arbitrarily defined as prediuresis, and aqueous excretion after feeding has stopped as diuresis.

In this report we will analyze prediuretic excretion quantitatively and compare it among 3 anopheline species of different subgenera and body size. At the same time, the results will be related to fecundity of Anopheles, and possible behavioral adaptations will be discussed. It is particularly interesting to compare our results with quantitative data reported for $A$ e. aegypti, the yellow fever mosquito (Briegel 1985).

\section{MATERIALS AND METHODS}

The following species were used for our experiments: Anopheles (Cellia) stephensi, An. (Nyssorhynchus) albimanus Wiedemann, and An. (Anopheles) quadrimaculatus Say. Larvae were reared on a highprotein diet (Lea 1964), with 300 larvae per pan in $450 \mathrm{ml}$ distilled water. The adults eclosed within a 2-day period and were of uniform size. They were kept continuously at $27^{\circ} \mathrm{C}, 85 \% \mathrm{RH}$, under longday conditions (16L:8D), with sunset and sunrise simulated by continuous dim light for $30 \mathrm{~min}$.

Experimental blood meals were given either in measured volumes by enema (Briegel \& Lea 1975) or by feeding the females individually on a restrained guinea pig until repletion, i.e., until withdrawal of the proboscis (generally between 1 and $10 \mathrm{~min}$ ).

For total nitrogen measurements, the excretory droplets were accumulated on a piece of cover slip held with forceps below the anus; evaporation was considered irrelevant for these determinations. For the hemoglobin determinations, however, a small glass vial containing $500 \mu \mathrm{l}$ of water was held near the anus to catch all rectal fluid released during feeding and to prevent denaturation of the he- 
moglobin. After the experimental blood meals, females were kept individually in reacting tubes $(10 \times 75 \mathrm{~mm})$. For the first $24 \mathrm{~h}$ they were kept without water; then they were provided with cotton pads soaked in $10 \%$ sucrose solution for the rest of the experiment. This combination kept mortality below $5-10 \%$. After 2 or 3 days the ovaries were dissected in saline and the mature oocytes counted; in the experiments on fecundity, ovaries were subsequently used for nitrogen determinations.

In several experiments the volume of the blood meal taken by feeding was estimated by weighing individual females before and after on a microbalance (Cahn DTL). For this purpose the females were anesthetized weakly with $\mathrm{CO}_{2}$; only those recovering instantly as indicated by avid biting activity were used for the experiments. Some of the females were used for midgut dissection to measure total nitrogen. Dissection was carried out in saline $(0.6 \%)$, and each midgut was analyzed individually after briefly washing it in a new drop of saline. Since the nitrogen content of the epithelium was below $2 \%$ of the blood bolus (unpubl. observ.), it was considered negligible.

Total nitrogen of the samples was determined through Nesslerization of a Kjeldahl digest (Minari \& Zilversmit 1963). For the hemoglobin determinations we used a Drabkin reagent (Briegel et al. 1978) calibrated with a standard human hemoglobin (525-18, Sigma Chemical Co., St. Louis, MO). In most experiments the same guinea pig was used as a host. The variations in its blood nitrogen or hemoglobin titers were averaged through periodic sampling by heart puncture, performed under anesthesia.

Linear regression lines for the data were computed using a commercially available program (HP$67)$.

\section{RESULTS}

Blood meal and simultaneous excretion of rectal fluid

Forty-eight An. stephensi females were fed individually to repletion on a restrained guinea pig. The total nitrogen was compared in (1) the pooled droplets of rectal fluid collected during feeding, (2) the blood in the midgut, and (3) a sample of blood from the host. The data and the linear regressions are presented in Fig. 1, where the midgut nitrogen and excretory nitrogen are each plotted against the nitrogen content of the complete

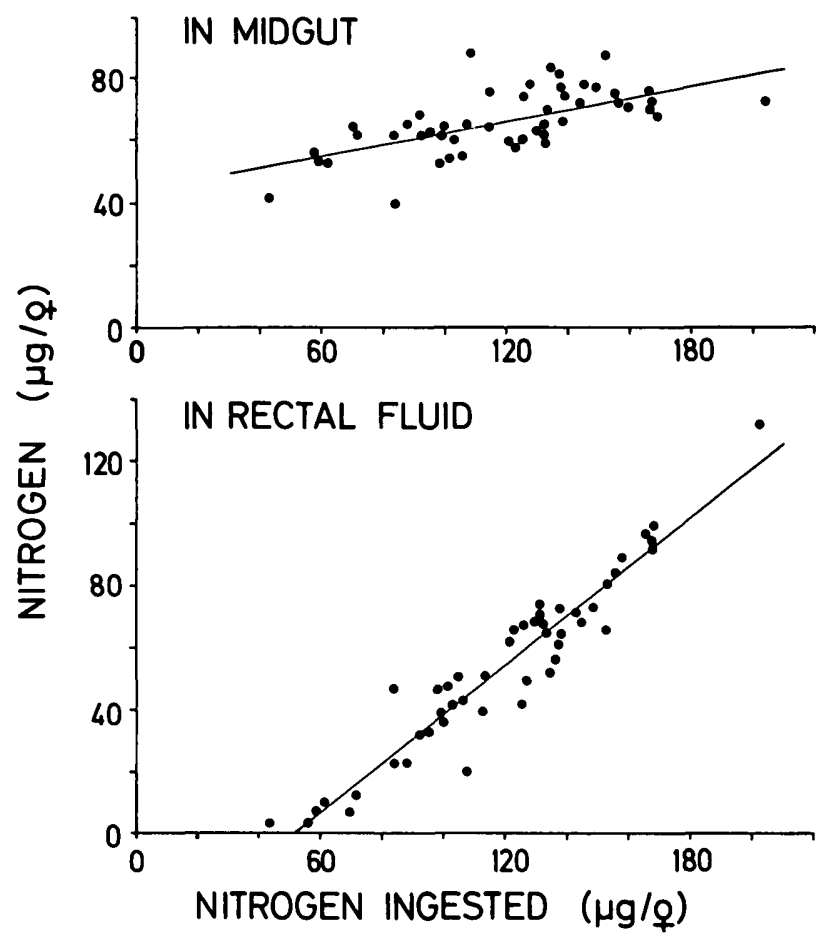

FIG. 1. Nitrogen retained in the midgut of An. stephensi or excreted with rectal fluid during feeding to repletion on a restrained guinea pig. Nitrogen ingested is the sum of the measurement from the midgut plus rectal fluid. Each point represents 19; linear regressions are given for midgut $(r=$ $0.661)$ and for rectal fluid $(r=0.958)$ from 48 .

blood meal (i.e., excretory plus intestinal nitrogen). We assumed that no substantial protein was degraded or absorbed during the feeding and collecting period. The maximal amount of intestinal nitrogen was about $80 \mu \mathrm{g} / \&$ (Fig. 1). This figure further indicates that rectal fluid was not released before 40 to $60 \mu \mathrm{g}$ of nitrogen had been retained in the midgut. Since the nitrogen concentration of the host blood was $19.82 \pm 1.44 \mu \mathrm{g} / \mu \mathrm{l}(n=5)$, the maximal sum of nitrogen (about $200 \mu \mathrm{g}$ ) indicated a blood consumption of up to $10 \mu \mathrm{l}$.

In Fig. 2 the midgut nitrogen (upper line) is compared with the volume of blood ingested, as determined by weight gain of these same females (lower line). Generally speaking, all midgut nitrogen measurements were consistently about $30 \mu \mathrm{g}$ higher than the extrapolation based on the weight increase and the host blood nitrogen (lower line). Thus blood meal nitrogen titers increased during feeding, and weighing the females underestimated the blood consumption substantially.

Total blood consumption per female was calculated by adding the average midgut and excretory nitrogen data and then dividing by the nitro- 


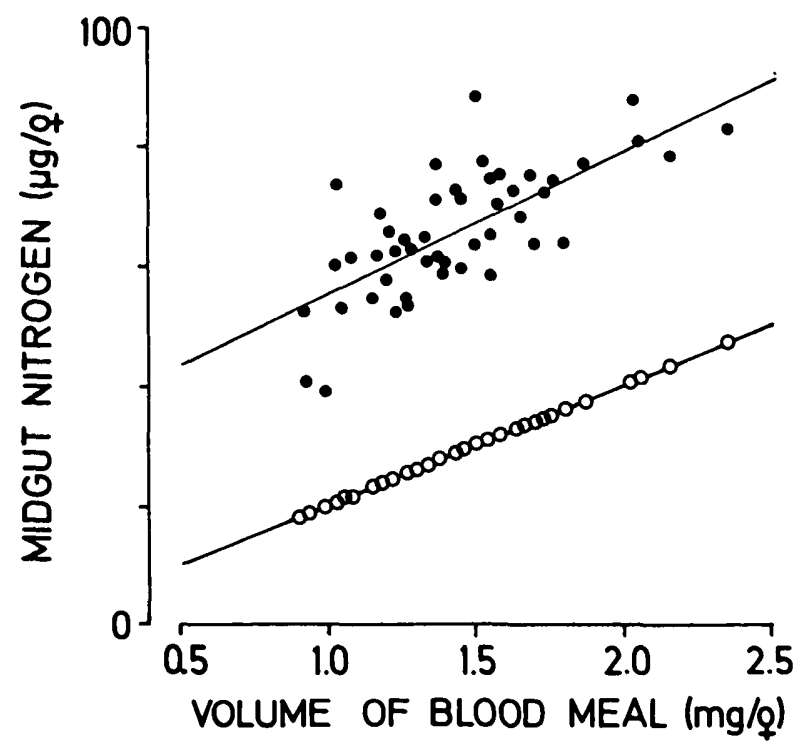

FIG. 2. Midgut nitrogen in An. stephensi after feeding to repletion on a guinea pig $(\bullet)$ and the amount of nitrogen ingested as calculated from the weight gains of 489 and the nitrogen content in the host blood $(0)$. Each point represents $1 \%$ and linear regressions are given $(r=0.72$ for $\theta ; r=0.96$ for O).

gen concentration in the host blood (Table 1). This revealed an average blood consumption of $6 \mu \mathrm{l} / \mathrm{q}$ An. stephensi, with individual variations between 2 and $10 \mu \mathrm{l}$.
Dividing the average midgut nitrogen by the weight of the blood (after its conversion to nitrogen) led to the concentration factor of 2.3. The loss of nitrogen through simultaneous excretion of rectal fluid varied tremendously between 2 and 130 $\mu \mathrm{g} / \%$; the average of $54 \mu \mathrm{g}$ was $45 \%$ of the total blood consumption.

When we gave measured amounts of blood by enema to $A n$. stephensi in $0.5-\mu$ l increments from 0.5 to $3 \mu \mathrm{l}$, we found that blood meals larger than $2 \mu$ l could never be injected without rupturing the midgut. Obviously, this quantity was the maximal volume of the distended abdominal midgut for this species reared under our conditions. This volume also coincided with the amount of blood retained in the midgut before the prediuretic excretion of rectal fluid began (see above). Relating this to the range of blood consumption of 2 to $10 \mu$ l (Table 1 ), it is evident that a substantial concentration of the blood protein took place in most females.

Since $80 \%$ of the vertebrate blood nitrogen occurs in the hemoglobin (Bursell 1965), and because of the red color of the rectal fluid, this experiment was repeated, this time measuring the hemoglobin in the pooled fluid, the midgut, and host blood samples. Exactly the same result was obtained (Fig. 3 ). At the end of feeding, the midgut contained an average of $499.9 \mu \mathrm{g}$ hemoglobin (range 313-676

TABLE 1. Comparison of the blood meal concentration among 3 anopheline species. All values are expressed as microlitres or were converted to micrograms of nitrogen, where applicable. $\mathrm{N}=$ nitrogen.*

\begin{tabular}{|c|c|c|c|c|c|c|}
\hline & \multicolumn{2}{|c|}{$\begin{array}{l}\text { An. stephensi } \\
(n=48 \%)\end{array}$} & \multicolumn{2}{|c|}{$\begin{array}{c}\text { An. albimanus } \\
(n=579)\end{array}$} & \multicolumn{2}{|c|}{$\begin{array}{c}\text { An. } \\
(n=44 a d r i m a c u l a t u s \\
(n=449\end{array}$} \\
\hline & $\mu \mathrm{\mu l} / \mathrm{q}$ & $\mu \mathrm{g} \mathrm{N}$ & $\mu \mathrm{l} / \mathrm{q}$ & $\mu \mathrm{g} \mathrm{N}$ & $\mu \mathrm{l} / q$ & $\mu \mathrm{g} \mathrm{N}$ \\
\hline \multicolumn{7}{|l|}{ Wt. of blood/\&** } \\
\hline $\begin{array}{l}\text { Mean (A) } \\
\text { Range }\end{array}$ & $\begin{array}{c}1.4 \\
0.9-2.4\end{array}$ & 28.5 & $\begin{array}{c}1.5 \\
0.6-2.8\end{array}$ & 29.3 & $\begin{array}{c}2.1 \\
1.3-3.4\end{array}$ & 40.6 \\
\hline \multicolumn{7}{|l|}{$\mathrm{N} /$ midgut } \\
\hline $\begin{array}{l}\text { Mean (B) } \\
\text { Range }\end{array}$ & & $\begin{array}{c}65.7 \\
38.9-87.1\end{array}$ & & $\begin{array}{c}54.7 \\
28.9-81.5\end{array}$ & & $\begin{array}{c}87.1 \\
58.9-123.9\end{array}$ \\
\hline \multicolumn{7}{|c|}{$\mathrm{N}$ excreted during feeding } \\
\hline $\begin{array}{l}\text { Mean (C) } \\
\text { Range }\end{array}$ & & $\begin{array}{c}53.8 \\
2.3-130.5\end{array}$ & & $\begin{array}{l}1.7 * * * \\
1.3-2.8\end{array}$ & & - \\
\hline \multicolumn{7}{|c|}{ Amt. of blood ingested/\& } \\
\hline $\begin{array}{l}\text { Mean }(B+C) \\
\text { Range }\end{array}$ & $\begin{array}{c}6.0 \\
2.2-10.2\end{array}$ & 119.5 & $\begin{array}{c}2.9 \\
1.5-4.2\end{array}$ & 56.4 & $\begin{array}{c}4.4 \\
3.0-6.3\end{array}$ & 87.4 \\
\hline \multicolumn{7}{|l|}{ Conc. factor } \\
\hline $\begin{array}{l}\text { Mean (B:A) } \\
\text { Range }\end{array}$ & & $\begin{array}{c}2.3 \\
1.4-3.1\end{array}$ & & $\begin{array}{c}1.9 \\
1.0-2.8\end{array}$ & & $\begin{array}{c}2.2 \\
1.5-3.1\end{array}$ \\
\hline
\end{tabular}

* All $\$ 8$ were fed on the same guinea pig with a nitrogen content of $19.82 \pm 1.44 \mu \mathrm{g} / \mu \mathrm{l}$ blood $(n=5)$.

** Increase of $\&$ fresh weight assumed to equal volume of blood retained in the midgut.

*** 98. 


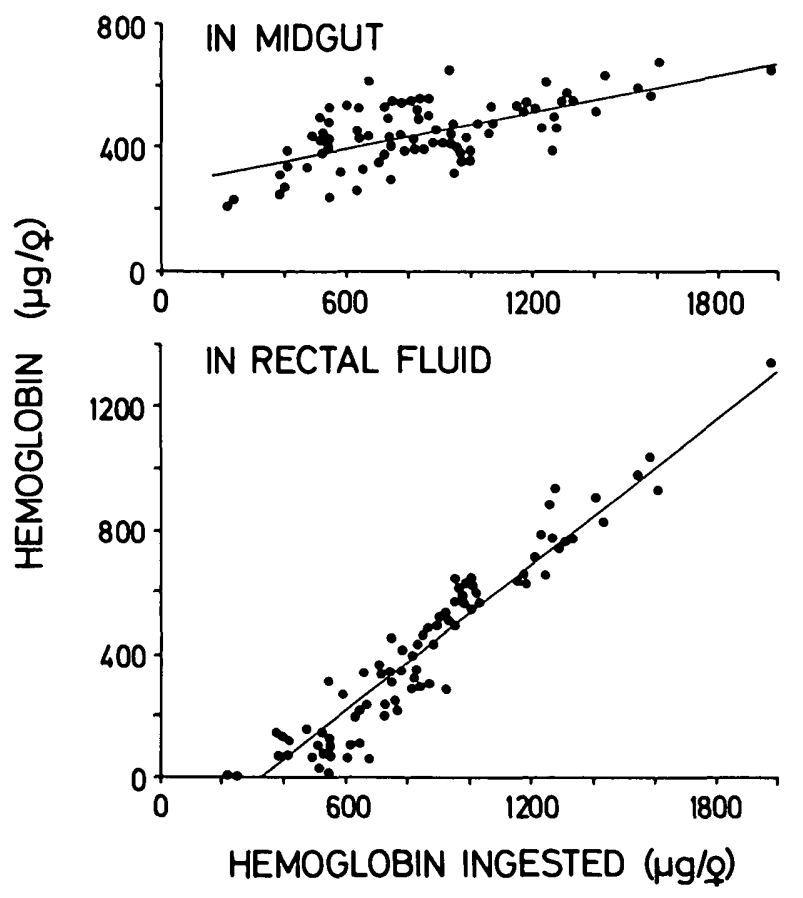

Fic. 3. Hemoglobin retained in the midgut of An. stephensi and excreted with rectal fluid during feeding to repletion on a restrained guinea pig. Hemoglobin ingested is the sum of the measurement from midgut plus rectal fluid. Each point represents 19; linear regressions are given for midgut $(r=$ $0.633)$ and for rectal fluid $(r=0.949)$ from 839 .

$\mu \mathrm{g}, n=30$ ), while in the corresponding samples of rectal fluid we detected an average of $407.5 \mu \mathrm{g}$ (range 12-930 $\mu \mathrm{g}$ ). Based on the hemoglobin titer in the host blood $(142.6 \pm 3.5 \mu \mathrm{g} / \mu \mathrm{l}, n=3)$, the average total hemoglobin ingested was roughly 6.5 $\mu \mathrm{l}$ of guinea pig blood (range $2.7-14.0 \mu \mathrm{l} / \%$ ), while only a volume of 1.4 to $2 \mu \mathrm{l}$ was retained in the midgut. When the midgut hemoblogin was compared to the values obtained by weighing the females (Fig. 4), it was ca. $300 \mu \mathrm{g}$ higher than the extrapolated values. Thus, the error in estimating blood ingestion by gravimetry is comparable to the one noted before with nitrogen determinations.

To prove that the red rectal fluid excreted by $A n$. stephensi during feeding represented excess blood, the following experiments were carried out. Giemsa-stained blood smears were prepared from the midgut contents immediately after feeding ended, as well as from the rectal fluid, and both were compared with smears from native host blood. Under the microscope, they all appeared identical, i.e., the rectal fluid contained intact erythrocytes, although at a much smaller density per optical field than the midgut preparation or the host blood (ca. $50 \%)$. Furthermore, at the end of feeding, the

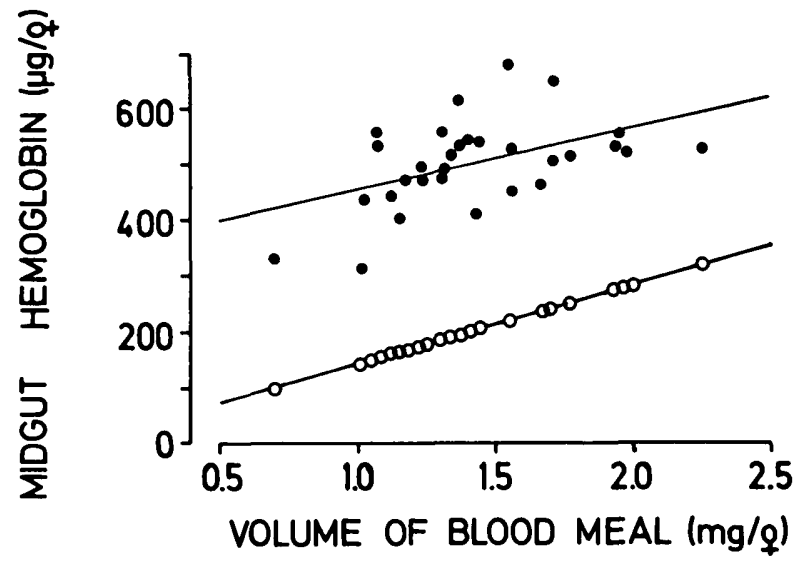

Fic. 4. Midgut hemoglobin in An. stephensi after feeding to repletion on a guinea pig $(\odot)$ and the amount of hemoglobin ingested as calculated from the weight gains of $30 \%$ and the content in the host blood $(O)$. Each point represents 19 and linear regressions are given $(r=0.47$ for $O ; r=1.00$ for $O$ ).

pooled rectal fluid, the entire midgut contents from the same female, and $2 \mu$ l of host blood were each subjected to an acid hydrolysis $\left(6 \mathrm{~N} \mathrm{HCl}, 130^{\circ} \mathrm{C}\right.$, $13 \mathrm{~h}$ ), and the molar distribution of the amino acids was compared by the percentage of their totals. All 3 samples had identical relative compositions, indicating the absence of selective absorption or retention of specific amino acids (or peptides or proteins) from the blood meal during feeding.

\section{Comparison with other Anopheles species}

Blood feeding and simultaneous prediuretic excretion in An. stephensi was compared with An. albimanus and An. quadrimaculatus. Since in both species the rectal fluid is clear, apparently lacking any hemoglobin, we measured only total nitrogen in the samples.

Fifty-seven An. albimanus were fed individually on the same guinea pig, and the samples were collected as before. The total nitrogen per midgut at the end of feeding averaged $54.7 \mu \mathrm{g}$ (Table 1 ), but only $1.7 \mu \mathrm{g} / \%(n=9)$ was recovered in the large volume of rectal fluid, corresponding to less than $5 \%$ of the midgut content. The actual blood consumption in this species was between 1.5 and 4.2 $\mu \mathrm{l} / \%$, with an average concentration factor of 1.9 .

Forty-four An. quadrimaculatus fed on the same guinea pig were treated and analyzed in the same way. The total nitrogen per midgut at the end of feeding was $87 \mu \mathrm{g}$, yielding an average blood consumption of $4.4 \mu \mathrm{l} / \mathrm{q}$; the concentration factor was 2.2 (Table 1). In this experiment the nitrogen excretion was not quantified, but comparing the midgut nitrogen with blood consumption indicated that 
TABle 2. Fecundity of An. stephensi after blood meal from guinea pig or man, administered by feeding on the host or by enema (mean $\pm \mathrm{SE}, n=$ no. determinations).

\begin{tabular}{ccccr}
\hline & AMT. OF & \multicolumn{3}{c}{ OVARIES } \\
\cline { 3 - 4 } BLOOD MEAL & $\begin{array}{c}\text { BLOOD IN } \\
\text { MIDGUT } \\
\text { AND HOST }\end{array}$ & $\begin{array}{c}\text { No. } \\
(\mathrm{mg} / 8)\end{array}$ & $\begin{array}{c}\text { NITROGEN } \\
\text { CONTENT } \\
(\mu \mathrm{g} / \text { ( })\end{array}$ & $n$ \\
\hline Feeding on & $0.7-1.0$ & $86 \pm 18$ & $13.5 \pm 3.0$ & 8 \\
guinea pig* & $1.0-1.5$ & $95 \pm 22$ & $16.8 \pm 3.4$ & 19 \\
& $1.5-2.0$ & $122 \pm 13$ & $21.7 \pm 0.9$ & 6 \\
& $2.0-2.2$ & $157 \pm 9$ & $26.4 \pm 3.0$ & 3 \\
Enema: guinea & 1.0 & 36 & 10.0 & 2 \\
pig blood* & 1.5 & $60 \pm 9$ & $14.2 \pm 1.4$ & 17 \\
& 2.0 & $88 \pm 14$ & $18.6 \pm 1.8$ & 15 \\
Enema: human & 1.0 & 0 & & 6 \\
blood** & 1.5 & $34 \pm 4$ & $8.2 \pm 1.2$ & 11 \\
& 2.0 & $50 \pm 12$ & $11.6 \pm 2.0$ & 13 \\
\hline
\end{tabular}

* Nitrogen content: $25.7 \pm 0.9 \mu \mathrm{g} / \mu \mathrm{l}(n=5)$.

** Nitrogen content: $33.4 \pm 1.8 \mu \mathrm{g} / \mu \mathrm{l}(n=5)$.

nitrogen excreted was less than $1 \%$ of the nitrogen ingested (Table 1).

Table 1 further demonstrates that the size range of blood meals ingested was related to the size of the mosquitoes. Anopheles quadrimaculatus, the largest species tested, ingested 1.3 to $3.4 \mathrm{mg}$ of blood ( $50 \%$ of all females between 1.6 and $2.2 \mathrm{mg}$ ), whereas $A n$. stephensi, the smallest species, retained only 0.9 to $2.4 \mathrm{mg}$ blood (50\% of all females between 1.2 and $1.6 \mathrm{mg}$ ). Conversely, the smallest species showed the highest consumption (2 to 10 $\mu l$ ), accompanied by the highest loss of nitrogen through prediuretic excretion, i.e., 2-131 $\mu \mathrm{g} /$ \%.

\section{Significance of blood meal concentration} for fecundity

To test the effect of prediuretic blood meal concentration on fecundity, $36 \mathrm{An}$. stephensi were fed to repletion on a guinea pig, while 34 other females were given enemas. In addition, 30 females were given human blood by enema. The number of mature oocytes was counted and compared to the midgut blood content and to the ovarian nitrogen (Table 2).

Fecundity was doubled when blood was taken by feeding as opposed to an enema of guinea pig blood. Enemas of human blood, on the other hand, led to a clearly reduced fecundity, despite its higher nitrogen content. Utilization of the dietary nitrogen was 47 to $51 \%$ with enemas of guinea pig blood, but only 16 to $17 \%$ with enemas of human blood. To our surprise, the average nitrogen content per egg varied considerably, depending on how the blood meal was acquired: lower after feeding (guinea pig: $0.17 \pm 0.02 \mu \mathrm{g} / \mathrm{egg}, n=36$ ), and significantly higher when given by enema (guinea pig: $0.23 \pm 0.03 \mu \mathrm{g} / \mathrm{egg}, n=34$; human: $0.24 \pm$ $0.03 \mu \mathrm{g} / \mathrm{egg}, n=24 ; P<0.001$ for both hosts).

To our knowledge, the usual practice of feeding Anopheles in the laboratory daily never had been subjected to a physiological analysis. Therefore, we tried to clarify whether females having fed once would actually refeed the following day, and whether multiple blood meals were required to complete 1 gonotrophic cycle. Of 25 An. stephensi fed to repletion on a guinea pig and thus containing a concentrated blood meal, $17(68 \%)$ refed $24 \mathrm{~h}$ later. In another experiment, the 1 st blood meals were given by enema $(0.5,1.0,1.5$, and $2.0 \mu \mathrm{l}$ of guinea pig blood). Twenty-four hours later these females were individually exposed to the guinea pig, and from $50 \%$ (initially given $2 \mu \mathrm{l}$ ) to $100 \%$ (given $0.5 \mu \mathrm{l}$ ) refed readily. Obviously, the lack of blood concentration owing to the enema technique did maintain biting activity.

In a similar experiment with An. albimanus, the 1 st meals were enemas of 0.5 to $3.0 \mu$ l guinea pig blood. None of the females receiving 2.5 or $3 \mu \mathrm{l}$ refed the next day; however, females initially given 0.5 to $1.5 \mu \mathrm{l}$ refed the following day, and even refed a 2nd time (i.e., $48 \mathrm{~h}$ after the enemas). Each of these blood meals led to an increase in the egg counts. For example, a female given only $0.5 \mu \mathrm{l}$ matured 17 eggs, while after a 2nd meal by feeding, her sister matured 82 eggs; a 3 rd meal by feeding produced 128 eggs. In a case with a $1.5-\mu$ l enema, the 3 rd meal produced 185 eggs, in contrast to 52 with the enema alone. Although these results are too scant for statistical analysis, they demonstrate convincingly that fecundity is considerably enhanced by multiple feedings.

\section{DISCUSSION}

In all 3 malaria vectors tested, the average nitrogen or hemoglobin content of the midgut at the end of feeding was at least double that determined by weighing females. This is the result of prediuretic excretion, i.e., excretion of serum or serum and erythrocytes as blood is ingested. Therefore, protein is accumulated substantially in the anopheline midgut, and the amount of protein available for oogenesis (Table 1, midgut) is comparable to that found in aedine mosquitoes (Briegel 1985). Since excretion starts during feeding as soon as the midgut is filled, it appears to be an overflow of blood from the distended midgut. The maximal volume that the abdominal midgut could hold 
without rupturing was found to be $2 \mu \mathrm{l}$ in $A n$. stephensi and $3 \mu \mathrm{l}$ in $A n$. albimanus, only $1 / 2$ or less of the amount observed in Ae. aegypti (6-7 $\mu$; Briegel 1985). Regular diuresis, initiated only after termination of feeding in Aedes (Boorman 1960, Stobbart 1977, Jones \& Brandt 1981, Williams et al. 1983) as well as in Anopheles (Nijhout \& Carrow 1978), serves primarily to reduce the flight weight of the newly fed female. The prediuretic excretion reported here, however, seems to be an additional adaptation evolved by these mosquitoes primarily to compensate for the smaller volume of the midgut and/or its limited elasticity. Indeed, it is followed by normal diuresis as reported in the literature.

There is, however, a considerable difference between $A n$. stephensi and the other 2 Anopheles tested: $A n$. albimanus and An. quadrimaculatus have an efficient "filtration system," producing a clear rectal fluid containing little or no nitrogen. This is in contrast to the rectal fluid of $A n$. stephensi, which contains erythrocytes. Could this be interpreted as a more primitive evolutionary stage than in other Anopheles, perhaps comparable to plant feeding in aphids, which concentrate valuable nutrients by passing excessive fluids?

There are certain disadvantages to this concentrating mechanism. Besides losing protein, the feeding time of $A n$. stephensi is prolonged considerably (up to $10 \mathrm{~min}$ or more) when compared to the other Anopheles species or to Aedes (feeding times 1-2 min). A behavioral correlate to this extended feeding might be the biting activity during dusk or night, as is often encountered among anophelines (Horsfall 1972) because reduced defensive behavior of sleeping hosts is likely.

So far it remains an open question as to why $A n$. stephensi cannot prevent the loss of protein. One possible explanation might be that the peritrophic membrane is formed much later in this than in other species (Freyvogel \& Stäubli 1965).

Accumulation of protein in the midgut through prediuretic excretion is clearly reflected by fecundity: the number of eggs produced per female Anopheles after ingesting a "full meal" is similar to or even higher than that in Ae. aegypti (Christophers 1960, Briegel 1985). The utilization of the blood meal nitrogen for oogenesis varied between 30 and $40 \%$ and was comparable to that in $A e$. aegypti. Fecundity was equally affected by the source of the blood as it is in Aedes: with human blood, fecundity was reduced by $1 / 2$ as compared to rodent blood, for the same reasons reported elsewhere
(Briegel 1985). Injecting blood meals by enema, on the other hand, also led to reduced fecundity as compared to feeding, in contrast to Aedes where no differences were observed between the 2 routes (Briegel 1985). This is explained by the absence of prediuretic concentration after enemas.

That in both An. stephensi and An. albimanus most females refed on a host $24 \mathrm{~h}$ after a previous blood meal points to 2 other basic differences compared to aedine mosquitoes. First, the humoral inhibition of another blood meal exerted by maturing oocytes (Klowden 1981) seems to be very weak or absent. No other time intervals have been tested yet, but in several cases even a 3rd meal was taken and utilized (see above). Second, as we have indicated before, these subsequent blood meals were utilized for vitellogenesis, which was initiated by the $1 \mathrm{st}$ meal. This observation points to different mechanisms regulating fecundity than in $A e$. aegypti, where oosorption was demonstrated to start about 8 to $12 \mathrm{~h}$ after the 1 st (small) blood meal (Lea et al. 1978).

Acknowledgments. We thank Mr S. Zaba and Mrs R. Haigis for technical assistance. The amino acid analyses were kindly performed by Dr P. Borner on an automatic amino acid analyzer. We appreciate the critical reading of the manuscript by Dr A. O. Lea and the advice given by the reviewers.

\section{LITERATURE CITED}

Boorman, J.P.T. 1960. Observations on the feeding habits of the mosquito Aedes (Stegomyia) aegypti (L.): the loss of fluid after a blood meal and the amount of blood taken during feeding. Ann. Trop. Med. Parasitol. 54: 8-14.

Briegel, H. 1985. Mosquito reproduction: incomplete utilization of the blood meal protein for oogenesis. J. Insect Physiol. 31: 15-21.

Briegel, H. \& A.O. Lea. 1975. Relationship between protein and proteolytic activity in the midgut of mosquitoes. J. Insect Physiol. 21: 1597-1604.

Briegel, H., A.O. Lea \& M.J. Klowden. 1978. Hemoglobinometry as a method for measuring blood meal sizes of mosquitoes (Diptera: Culicidae). J. Med. Entomol. 15: 235-38.

Bursell, E. 1965. Nitrogenous waste products of the tsetse fly, Glossina morsitans. J. Insect Physiol. 11: 993-1001.

Christophers, S.R. 1960. Aedes aegypti (L.), the yellow fever mosquito. Cambridge University Press, Cambridge. 739 p.

Freyvogel, T.A. \& W. Stäubli. 1965. The formation of the peritrophic membrane in Culicidae. Acta Trop. 22: 118-47.

Gee, J. D. 1975. The control of diuresis in the tsetse fly Glossina austeni: preliminary investigation of the diuretic hormone. J. Exp. Biol. 63: 391-401.

Gerberg, E. J. 1970. Manual for mosquito rearing and experimental techniques. American Mosquito Control Assoc., Selma, CA. 109 p.

Horsfall, W. R. 1972. Mosquitoes, their bionomics and relation to disease. Hafner Publishing Co., New York. 723 p.

Jones, J.C. \& E. Brandt. 1981. Fluid excretion by adult Aedes aegypti mosquitoes. J. Insect Physiol. 27: 545-49.

Klowden, M. J. 1981. Initiation and termination of host-seek- 
ing inhibition in Aedes aegypti during oocyte maturation. $J$. Insect Physiol. 27: 799-803.

Lea, A.O. 1964. Studies on the dietary and endocrine regulation of autogenous reproduction in Aedes taeniorhynchus (Wied.). J. Med. Entomol. 1: 40-44.

Lea, A.O., H. Briegel \& H.M. Lea. 1978. Arrest, resorption, or maturation of oocytes in Aedes aegypti: dependence on the quantity of blood and the interval between blood meals. Physiol. Entomol. 3: 309-16.

Maddrell, S.H.P. 1964. Excretion in the blood-sucking bug, Rhodnius prolixus Stål. II. The normal course of diuresis and the effect of temperature. J. Exp. Biol. 41: 163-76.

Minari, O. \& D.B. Zilversmit. 1963. Use of KCN for stabili- zation of color in direct Nesslerization of Kjeldahl digests. Anal. Biochem. 6: 320-27.

Nijhout, H.F. \& G.M. Carrow. 1978. Diuresis after a blood meal in female Anopheles freeborni. J. Insect Physiol. 24:29398.

Stobbart, R.H. 1977. The control of the diuresis following a blood meal in females of the yellow fever mosquito Aedes aegypti (L.). J. Exp. Biol. 69: 53-85.

Williams, J.C., H.H. Hagedorn \& K.W. Beyenbach. 1983. Dynamic changes in flow rate and composition of urine during post-bloodmeal diuresis in Aedes aegypti (L.).J. Comp. Physiol. 153: 257-65. 